\title{
Cross-comparative Analysis of Frequentist and Bayesian Perspectives on Failure Time Distributions
}

\author{
Anthony Joe Turkson \\ Mathematics, Statistics and Actuarial Science Department, Takoradi Technical University, Sekondi- \\ Takoradi, Ghana \\ e-mail: anthony.turkson@ttu.edu.gh
}

\begin{abstract}
In reliability analysis, both the Weibull and the lognormal distributions could be analyzed using the observed data logarithms. While the Weibull data logarithm is skewed, the lognormal data logarithm is symmetrical. This review work initiates discussions on and syntheses of the various views held on the use of the frequentist and Bayesian approaches to drawing statistical inferences on failure time distributions of survival models. Of greater concern was the discussion on the use of the exponential, Weibull and log-normal distributions in reliability analysis. Various methods have been used to discriminate between the two most important distributions. They include: Coefficients of variation (CV); the standard deviation of the data logarithms (SD); the percentile position of the mean of the data logarithm (PP); the cumulated logarithm dispersion before and after the mean (CLD); ratio of the maximum likelihood (RML); Kullback-Leibler Divergence (KLD); and Minimized Kullback-Leibler Divergence (RMKLD). In the (CV, SD, PP and CLD) study, a stress-strength data set was used for the analysis. The stress data followed a lognormal distribution, while the strength data followed a Weibull distribution, therefore for the stress-strength analysis the lognormal-Weibull combination was used. In the ratio of the maximum likelihood (RML) study, it was averred that though each of the distributions had great applications, none of them produced a good fit. In the study using Kullback-Leibler Divergence (KLD); and Minimized Kullback-Leibler Divergence (RMKLD), test results revealed that $R M L=0.345>0$. Hence the lognormal distribution was selected. Similarly, the $R M K L D=0.6028>0$, therefore the Weibull distribution was selected. In the final analysis, none of the study results could reject the Weibull in favour of the lognormal distribution model. In respect of the frequentist and the Bayesian approach to conducting statistical inferences, it came out strongly that it was
\end{abstract}

Received: January 4, 2022; Accepted: February 12, 2022

2010 Mathematics Subject Classification: 62H17.

Keywords and phrases: Bayesian, exponential, frequentist, log-normal, Weibull distribution. 
high time psychologist who had adopted the use of the frequentist framework moved away from it and started using the Bayesian approach. A shift towards the use of the Bayesian, lognormal-Weibull approach is thus recommendable.

\section{Introduction}

Failure time analysis (FTA) addresses data in the form "time until an event of interest occurs". In their attempt to describe the nature, timing and occurrence of events, biostatisticians have turned their attention to the use of survival data analytic techniques alias (FTA). Survival and hazard functions have distinguished themselves as very effective and influential statistical tools used in describing the probability distributions associated with the timing and occurrence of events. However, because different processes generate different patterns of survival data, many distributions are being used for modeling the distribution of failure times. In using parametric methods of estimation, one of the key issues that need to be addressed is how to choose a probability distribution that best describes the underlying processes that generated the observed data. One key reason for selecting the best fitting survival distribution is because the model it represents will be used to make key decisions. One cannot just use the exponential distribution because it is the simplest, neither can they use the either the lognormal or Weibull distribution because they appear to be extensively used and robust. The use of incorrect models especially in reliability analysis can lead to serious problems such as damage of expensive equipment, premature failures of products and the like which may result in customer dissatisfaction. Some critical questions that ought to be addressed are: Does the postulated exponential probability distribution accurately represent the relationship between the time and the likelihood of survival? Is the exponential model useful? Is the hazard rate actually constant? How can one determine the Weibull parameters from data? How can one determine Weibull and lognormal parameters from data by maximum likelihood estimates? These are all legitimate questions which need to be addressed.

The best way to prevent possible modeling errors, is to develop more valid models, make better decisions, and apply cutting-edge probability distribution fittings. This technique allows researchers to select the probability distribution which best describes the reliability of a component or system, based on available historical data. However, the use of distribution fitting is connected with complex calculations which require special knowledge in the field of statistics or programming. The problem of selecting the best fitting distribution can be easily solved by applying the specialized distribution fitting software Easy-Fit (https://www.xlstat.com/en/solutions/features/easy-fit). This software 
product is designed to automate the whole distribution fitting process. It performs all the calculations for the researcher, the researcher only has to interpret the results and select the best model. A school of thought was of the view that postulating that survival times were sampled from a population with a constant hazard rate was undoubtedly unrealistic in many situations [1]. In addition, [1] underscored the fact that the shape and scale parameters of the Weibull distribution provide increased flexibility that potentially improves the description of collected survival time data.

In this paper I looked at the exponential, two-parameter and three parameter Weibull, and lognormal distributions. My goal was to bring to bear my knowledge in the area juxtaposing it with the stock of knowledge from other researchers. Though I do not see myself as an expert in fitting survival data, I believe the intensive research I have done in this area could serve as a knowledge breather to other researchers. This paper sought to provide a comprehensive and cross comparative review of three of the probability distributions used in fitting survival data namely: exponential; Weibull; and log-normal probability distributions, simulation plots using the $\mathrm{R}$ platform which were meant to reveal the graphical fit of the distributions were run; the topic was addressed from both a frequentist and Bayesian perspective.

\subsection{Frequentist and Bayesian approach}

Researches are indicative of the fact that the frequentist methods treat model parameters as unknown, having fixed constants and employing only observed data to estimate the values of parameters. With respect to failure time data, we may as well assume the data to be exponentially distributed:

$$
f(x ; \lambda)=\frac{1}{\lambda} e^{-\frac{x}{\lambda}}, \text { for } x \geq 0,
$$

where $\lambda$ is the parameter of interest. Mean time between failures (MTBF) could be estimated as the total test time divided by the number of failures. The maximum likelihood estimator for $\lambda$ in this equation is simply the total test time divided by the number of failures. This provides a point estimate for MTBF. To describe the variability in the estimate, a confidence interval could be calculated [2]. With a small data set or in the event that no failures were observed, the bounds could be wide and minimally informative. In the event that there were no failures, point estimates would be unavailable. It is worthy of note that confidence bounds do not describe the range of values the parameter occupies, but describes the uncertainty associated with a sampling method [3]. 
Researchers like [4-10] have underscored the fact that the following merits and demerits accrue to the frequentist inference, that the method: Is based on the idea that probability is a limiting frequency; does not allow probability statements about the parameters of a statistical process; is less-than-ideal method for statistical inference; does not condition on the observed data; depends on data that were never observed; depends on the intention with which the data were collected; does not prescribe which estimator is best; does not quantify statistical evidence; does not apply to non-nested models; and is not as objective as one may think. It can therefore be concluded from the points raised that the frequentist procedures suffer from fundamental philosophical and practical problems.

Researchers are apt in asserting that the Bayesian methods treat parameters as unknown random variables whose distribution (the prior) represents the current belief about the parameter. In respect of that, the Bayesian method calculates probabilities associated with the parameters, given the data. An introspective view of the Bayes' rule defined as Equation (1) reveals four important attributes:

$$
P(\beta \mid \mathrm{D})=\frac{P(D \mid \beta) P(\beta)}{P(D)},
$$

where,

a. $P(\beta \mid D)$ is the posterior: Probability distribution of the parameter $(\beta)$ given a data set (D)

b. $P(D \mid \beta)$ is the likelihood: A function of the observed data $(D)$ given a parameter $(\beta)$

c. $P(\beta)$ is the prior: Probability distribution of the parameter $(\beta)$ which represents our belief on the parameter before the dataset $\mathrm{D}$ is observed

d. $P(D)$ is the evidence: Probability distribution of the observed data $(D)$ which acts as a normalizing constant that ensures that the cumulative posterior distribution sums up to one(1).

Our understanding of the maximum likelihood estimate (MLE) is that, it is used when one wants to find the parameter values that best fit the dataset using a specified distribution. The likelihood term in the specifications represents this type of information. The difference is that the likelihood and prior are inputs to Bayesian analysis, not the output. The critical point in Bayesian analysis is that the posterior is a probability 
distribution function (pdf) of the parameter given the data set, not simply a point estimate. This enables all the properties of a pdf to be employed in the analysis.

The researchers [4-10] equally underscored the fact that the following merits and demerits accrue to the Bayesian inference: parameters are random variables; degree of belief with respect to the parameters is quantified by probability distributions; inference is prescriptive; given the specification of a model, there exists only one way to obtain the appropriate answer; captures all of the replicable structure and ignores all of the idiosyncratic noise; does not make a fundamental distinction between nested and nonnested models; allows for the flexible implementation of relatively complicated statistical techniques such as those that involve hierarchical nonlinear models; makes it easier to focus on the relevant variables by integrating out so-called nuisance variables; yields result that connect closely to what researchers want to know; is subjective and therefore has no place in scientific communication; in hypothesis testing, it allows one to monitor the evidence as the data come in; and it allows prior knowledge to influence conclusions.

\subsection{Exponential probability distribution}

In Survival analysis, if the product-limit estimate of the distribution is model free, then the resulting estimates are not dependent on any assumptions, moreover, it would not require that knowledge about the population that produced the sampled survival data be known. This property is inherent in the exponential distribution. This distribution happens to be a simple but useful theoretical distribution that completely defines a survival probability based on a single parameter. The exponential distribution is one of the widely used continuous distributions, it is used mostly to model the time between events. Statistically, it is used to model Poisson processes, in which case, an object which was initially in state ' $A$ ' changes to state ' $B$ ' with constant probability per unit time $\operatorname{lambda}(\lambda)$, which is the time at which the state changes. Though the exponential distribution is the simplest, it is the most widely used distribution in survival studies [11]. The exponential distribution is characterized by a single parameter $\lambda$ and it has a constant hazard function over $T$. The failure rate is independent of $t$ and the chance of failure over any given time interval is the same. In the literature, this property is referred to as the 'lack of memory' property, in that, time does not affect survival [11,12]. Small (large) values of $\lambda$ indicate low (high) risk and long (short) survival [11]. Exponential distribution is extensively used in reliability engineering to describe units that have a constant failure rate. It is also used to model the time between events that happen at a constant average rate e.g., the time until the next phone call arrives, the time until a 
radioactive particle decays, time until reinfection of a common disease, and the interarrival times. The loss of electrical charge, the oscillations of a spring, the physics of heat loss from an object, interest accumulated in a bank account are also situations where this distribution is applied. Because time does not affect failure, the occurrence of the event is random.

Mathematically, the exponential distribution is defined as follows. If $T$ is a continuous random variable, then $T$ is said to have an exponential distribution with parameter $\lambda>0$, written as $T \sim \operatorname{Exponential}(\lambda)$. With the probability density function (pdf) given by:

$$
f_{T}(t)=\left\{\begin{array}{l}
\lambda e^{-\lambda t} \quad t>0 \\
0 \quad \text { otherwise }
\end{array}\right.
$$

The cumulative density function (cdf) is obtained as follows: For $T>0$, we have

$$
F_{T}(t)=\int_{0}^{t} \lambda e^{-\lambda t} d t=1-e^{-\lambda t}
$$

Finding the expectation of $T, E(T)$ :

$$
\begin{gathered}
E(T)=\int_{0}^{\infty} t \lambda e^{-\lambda t} d t \text {, using the integration by parts method with } y=\lambda t, \\
E(T)=\frac{1}{\lambda} .
\end{gathered}
$$

Finding the variance of $T, V(T)$ :

$$
\begin{aligned}
E\left(T^{2}\right) & =\int_{0}^{\infty} t^{2} \lambda e^{-\lambda t} d t, \text { using integration by parts with } y=\lambda t, \\
& =\frac{1}{\lambda^{2}} \int_{0}^{\infty} y^{2} e^{-y} d y, \\
& =\frac{2}{\lambda^{3}} .
\end{aligned}
$$

From Equations (4) and (5), we obtain the following:

$$
\begin{aligned}
V(T) & =E\left(T^{2}\right)-(E T)^{2}, \\
& =\frac{2}{\lambda^{2}}-\frac{1}{\lambda^{2}}=\frac{1}{\lambda^{2}} .
\end{aligned}
$$

Other relevant functions are given below:

Hazard function: $h(t)=\lambda, \lambda>0, t \geq 0$.

Survival function: $S(t)=P(T \geq t)=e^{-\lambda t}, t \geq 0$. 
In this distribution, the relationship arises when the rate of change is proportional only to the size of the quantity it is changing.

The property that the exponential hazard function is constant follows directly from the general definition of a hazard rate,

$$
h(t)=-\frac{\frac{d}{d t} S(t)}{S(t)}=\frac{\frac{d}{d t} e^{-\lambda t}}{e^{-\lambda t}}=\frac{\lambda e^{-\lambda t}}{e^{-\lambda t}}=\lambda .
$$

A justification of the relationship between $S(t)$ and $h(t)$ follows:

The value of the expression $\left(1+\frac{1}{n}\right)^{n}$ is approximately equal to 2.714 for $n$ in the neighborhood of 200, when $n$ becomes infinite the value of the expression is denoted by $e$. The value of $e$ has been calculated to many non repeating decimal places as $2.71828182846 \ldots$. Furthermore the more general expression, $\left(1-\frac{x}{n}\right)^{n}=e^{-x}$ is also approximate for large $n$ and exact for infinitely large $n$, suppose a time interval $(o, t)$ is divided into $n$ equal subintervals of length $\delta(n \delta=t)$ and the conditional probability of surviving each subinterval is exactly the same $p_{i}=p$ for all $n$ subintervals. Then the survival probability is approximately.

$$
\begin{gathered}
S(t)=P(T \geq t)=\prod_{i=1}^{n} p_{i}=P^{n}=(1-q)^{n} . \\
\left(1-\frac{n q}{n}\right)^{n} \cong e^{-q n}=e^{-\lambda \delta n}=e^{-\lambda t} .
\end{gathered}
$$

Because the hazard rate $\lambda=q / \delta$ is constant, $(q=1-p)$ is constant for all subintervals and $\delta n=t$. The survival probability $S(t)$ becomes exactly $e^{-\lambda t}$ when $n$ is infinitely large, which is another way of saying that the exponential survival distribution becomes $S(t)=e^{-\lambda t}$ when the time $t$ is a continuous variable $\delta=0$.

Due to simplicity, the exponential distribution was the first widely used distribution model but the restrictiveness of a constant hazard function has led to cautious use in recent years [13]. For time $t=10$ years and $\lambda=0.02$, the survival probability:

$$
P(T \geq t)=S(t)=e^{-\lambda t}, t \geq 0
$$

becomes

$$
P(T \geq 10)=S(10)=e^{-0.02 \times 10}=0.82 .
$$


thus, for a randomly sampled item whose survival time is described by the exponential survival time, the probability of surviving past $t=10$ years is 0.82 .

\subsubsection{Application of the exponential function}

Illustration 1. The time until we receive the next phone call. At a call center, the average number of minutes it takes a receptionist to receives a new call is 20 minutes. To find the probability that a new customer calls within: a. $t=20$ to $t=30$ minutes. b. After $t=25$ minutes.

We proceed with the solution as follows. The average time between calls $\mu=20$ minutes. Thus, the rate can be calculated as: $\lambda=\frac{1}{\mu} \Rightarrow \lambda=\frac{1}{20}=0.05$. With the help of the EXPON.DIST $(t, \lambda$, Cumulative $)$ function in excel we get the following syntax:

a. $=$ EXPON. DIST(30, 0.05, True)- EXPON.DIST(20, 0.05, True) $=0.776-632=0.144$

b. $=$ EXPON. DIST $(25,0.05$, True $)=0.713$

Illustration 2. Time until cancer death. In the study of epidemiology, it was noted that the time it takes for every patient diagnosed of cancer to die in a certain region was 40 weeks on the average. After a recent diagnosis of a cancer patient, find the probability that it will take more than 50 weeks for the death to occur. We proceed with the solution as follows $\mu=40$ weeks. $\lambda=\frac{1}{\mu} \Rightarrow \lambda=\frac{1}{40}=0.025$. From the excel, we get the following syntax $=$ EXPON.DIST $(50,0.025$, True $)=0.713$

\subsubsection{The lack of memory property}

The memoryless (forgetfulness) property articulates that a given probability distribution is independent of its history, in other words, the past has no bearing on its future behavior. If a probability distribution has the memoryless property the likelihood of something happening in the future has no relation to whether or not it has happened in the past. The exponential distribution is the only memoryless continuous random variable [14].

Theorem. If $T$ is exponential with parameter $\lambda>0$, then $T$ is a memoryless random variable. That is,

$$
P(T>t+s \mid T>s)=P(T>t), \text { for } s, t \geq 0 .
$$


Proof.

$$
\begin{aligned}
P(T>t+s \mid T>s) & =P(T>t)=\frac{P(T>t+s, T>s}{P(T>s)} \\
& =\frac{P(T>t+s)}{P(T>s)} \\
& =\frac{1-F_{T}(t+s)}{1-F_{T}(s)} \\
& =\frac{e^{-\lambda(t+s)}}{e^{-\lambda(s)}}=e^{-\lambda(t)}=P(T>t) .
\end{aligned}
$$

The above property will be illustrated with an example. The probability that one survives an illness from study time $t=t_{0}$ to $t=t_{2}$, could be broken down into two sub sets: $t=t_{0}$ to $t=t_{1}$ and $t=t_{1}$ to $t=t_{2}$. In survival probability terms, probability of survival beyond $t=t_{2}$ is given by:

$$
\left.S\left(t_{2}\right)=P\left(T \geq t_{2}\right)=P\left(T \geq t_{2}\right) \mid T \geq t_{1}\right) \times P\left(T \geq t_{1}\right) .
$$

Resorting to Equation (8): $S(t)=P(T \geq t)=e^{-\lambda t}, t \geq 0$.

Equation (12) can be written as: $S\left(t_{2}\right)=e^{-\lambda t_{2}}=P\left(T \geq t_{2} \mid T \geq t_{1}\right) \times e^{-\lambda t_{1}}$.

For the interval $t=t_{1}$ to $t=t_{2}$, the conditional survival probability is,

$$
P\left(T \geq t_{2} \mid T \geq t_{1}\right)=e^{-\lambda\left(t_{2-} t_{1}\right)} .
$$

From Equation (14), we infer a key property of the exponentially distributed survival times, that is to say, the probability of surviving from $t=t_{1}$ to $t=t_{2}$ depends only on the magnitude of the difference between $t_{1}$ and $t_{2}$ and not the actual values of $t_{1}$ and $t_{2}$. From Equation (10), we note that if $t_{2}-t_{1}=10$ and $\lambda=0.02$, the survival probability $P\left(T \geq t_{2} \mid T \geq t_{1}\right)=e^{-\lambda\left(t_{2}-t_{1}\right)}$, becomes $P\left(T \geq t_{2} \mid T \geq t_{1}\right)=e^{-0,02(10)}=0.82$. The same results apply to the following two cases: $t_{2}=70, t_{1}=60$ and $t_{2}=30, t_{1}=20$, with the constant $\lambda=0.02$. Thus the probability of surviving 10 additional years is the same for 60-year-olds as it is for 20-year-olds. In a nut shell, we will say that the exponential survival distribution function describes the risk for situations that do not age or objects that do not succumb to tear and wear. Few examples in real life that follow the exponential survival distribution are as follows: Computer chips do not wear out irrespective of the number of years it has been used; the breaking of a drinking glass does not depend on how long it has been used or not used or how many times it has been used for drinking related purposes. 


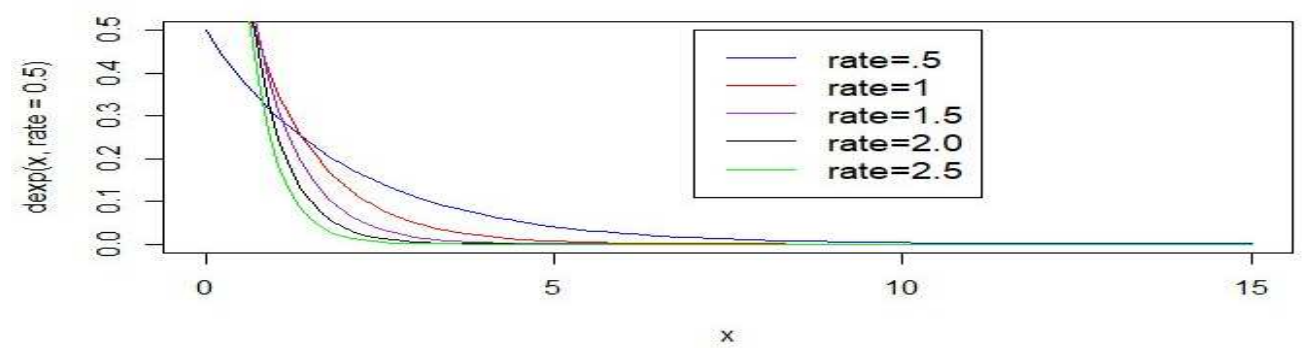

Figure 1: A plot of the exponential distribution. Using the $\mathrm{R}$ codes for different values of rate parameter.

\subsection{Weibull probability distribution}

In survival analysis modelling, the Weibull distribution has clinched an envious position as the most crucial distribution in modelling life time data. The distribution is a generalization of the exponential distribution that allows for a power dependence of the hazard on time. It has broader applications than the exponential distribution because it does not assume a constant hazard rate [11]. The Weibull distribution is one of the most commonly used distributions in reliability engineering. It is probably the most widely used distribution and is often used to test the life of manufactured items [13,15]. To the question: "Why do we use Weibull Distribution?" The answer is not farfetched; we use Weibull distribution for life data analysis for an item because of its flexibility to adapt to various conditions. Weibull analysis involves using the Weibull distribution and sometimes, the lognormal distribution to study lifetime data analysis. The Weibull analysis can be used to study the following: Lifetimes of medical and dental implants; components produced in a factory (bearings, capacitors or dielectrics); warranty analysis, utility services, and other areas where time-to-failure is important. The analysis is not limited to only production; it is also applicable to the design stage and in-service time as well. Due to technology and software programmes, the use of the Weibull distribution has been made simpler. The merits of the Weibull analysis are that it could be used for analyzing lifetime data with very small sample sizes and produces an easy-to-understandand-interpret plots.

The Weibull distribution is the only parametric regression model which has both a proportional hazards representation and an accelerated failure time representation. The relevant functions for the distribution are given below:

Survival function: $S(t)=P(T \geq t)=e^{-\alpha t^{\beta}}, t \geq 0 ; \alpha, \beta>0$. 
Hazard function: $h(t)=\frac{\frac{d}{d t} S(t)}{S(t)}=\frac{\alpha \beta t^{\beta-1} e^{-\alpha t^{\beta}}}{e^{-\alpha t^{\beta}}}=\alpha \beta t^{\beta-1}$.

Probability density function: $f(t)=\alpha \beta\left(\alpha t^{\beta-1}\right) e^{-\alpha t^{\beta}}$.

Cumulative functions: $F(t)=1-e^{-\alpha t^{\beta}}$.

The two-parameter Weibull distribution is presented as follows:

$$
f(t ; \alpha, \beta)=\frac{\beta}{\alpha}\left(\frac{t}{\alpha}\right)^{\beta-1} \exp -\left(\frac{t}{\alpha}\right)^{\alpha}, t \geq 0 ; \alpha, \beta>0
$$

$\beta$ controls the shape of the distribution and is called the shape parameter, while $\alpha$ is known as the scale parameter. The failure rate function is given by the equation:

$$
h(t ; \alpha, \beta)=\frac{\beta}{\alpha}\left(\frac{t}{\alpha}\right)^{\beta-1} .
$$

Equation (20) is a monotonic decreasing function in $t$ if $\beta<1$, and a monotonic increasing function in $t$ if $\beta>1$. It reduces to the exponential distribution when $\beta=1$ [12]. The Weibull distribution is often used to model the time until a given technical device fails, if $\beta<1$, then the failure rate of the device decreases over time; if $\beta=1$, then the failure rate of the device is constant over time; if $\beta>1$, then the failure rate of the device increases over time. Impliedly the distribution can be used to model survival distributions of populations with increasing, decreasing, and constant risk [11]. As has been mentioned earlier, the Weibull distribution is characterized by the two parameters, $\alpha(\alpha \geq 0)$ and $\beta(\beta \geq 0)$. The shape parameter $\beta$ determines the shape of the distribution $[11,13]$. While $\alpha$ determines the scale of the distribution [11].

If $T$ follows the generalized Weibull distribution, then the following distributions can be generated.

Survival function: $S(t)=P(T \geq t)=1-\left(1-e^{-\alpha t^{\beta}}\right)^{\lambda}, t \geq 0 ; \alpha, \beta, \lambda>0$.

Cumulative Hazard rate function: $H(t)=-\ln \left\{1-\left(1-e^{-\alpha t^{\beta}}\right)^{\lambda}\right\}$.

Hazard rate function: $h(t)=\frac{\lambda \alpha \beta t^{\beta-1} e^{-\alpha t^{\beta}}\left(1-e^{-\alpha t^{\beta}}\right)^{\lambda-1}}{1-\left(1-e^{-\alpha t^{\beta}}\right)^{\lambda}}$.

With respect to Equations (21), (22) and (23), the following six (6) conditions apply.

Conditionalities of the shape parameter $\beta$ and location parameter $\lambda$ 
a) If $\beta=1, \lambda=1$, the failure rate is constant, that is, it becomes an exponential function.

b) If $\beta \neq 1, \lambda=1$, the failure rate is monotonic Weibull.

c) If $\beta<1, \lambda<1$, the failure rate is decreasing Weibull.

d) If $\beta>1, \lambda>1$, the failure rate is increasing Weibull.

e) If $\beta>1, \lambda<1$, the failure rate is bathtub or increasing Weibull.

f) If $\beta\langle 1, \lambda\rangle 1$, the failure rate is unimodal or decreasing Weibull.

From the conditionalities above, it is note that the generalized Weibull family is versatile.

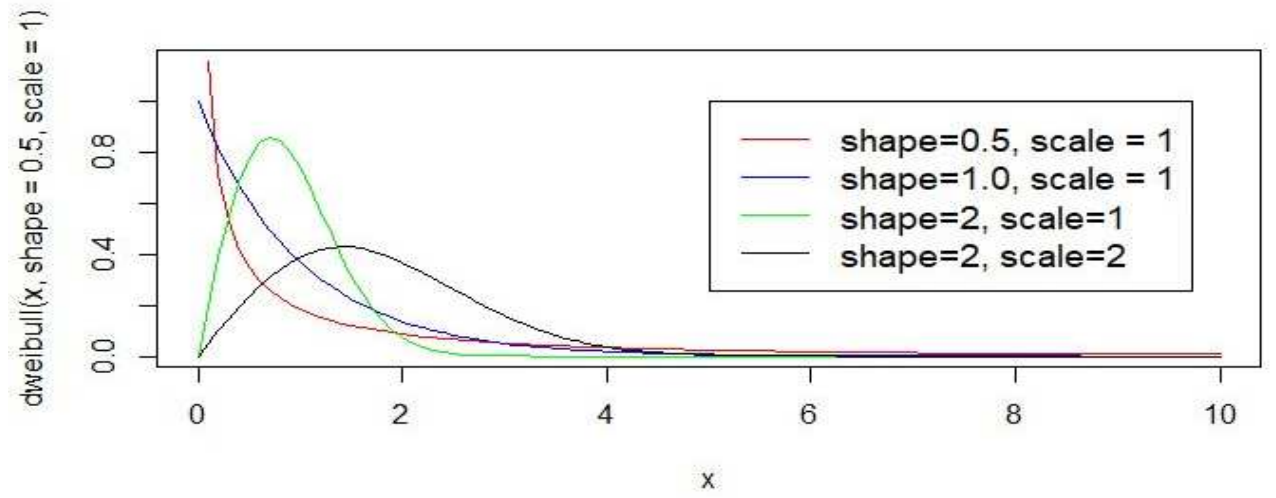

Figure 2: The horizontal axis on a Weibull plot shows lifetimes or aging parameters like mileage, operating times, or cycles of use. The vertical axis shows cumulative (additive total) percentage failures. The shape parameter varied while the scale was kept constant plus scale parameter varied while shape parameter was kept constant.

\subsection{Log-normal probability distribution}

The Lognormal distribution is based on the Normal distribution. A random variable is log normally distributed if the logarithm of the random variable is normally distributed. In other words, the log-normal distribution is a distribution of a variable whose logarithm follows the normal distribution [11]. The Lognormal distribution has widespread application. The distribution is used in situations where values are positively skewed. The distribution is applied in financial analysis for security valuation or in real estate for property valuation. It is extensively used in reliability analysis. It can also be used to model the long-term return rate on a stock investment, the weight and blood pressure of 
humans and the survival time of bacteria in disinfectants. To the question: Why is the lognormal distribution used so extensively? The response is that, it is multifaceted with implications in the field of finance, economics and engineering and so on. The distribution assumes that if $Y=\log T$ is normally distributed with mean $\mu$ and variance $\sigma^{2}$, then, the lifetime $T$ is log-normally distributed with scale parameter $\mu$ and shape parameter $\sigma^{2}$, (written as $T=\Lambda\left(\mu, \sigma^{2}\right)$. And the probability density function of $X$ is:

$$
\frac{1}{\sigma \sqrt{2 \pi}} e^{-\frac{1}{2}\left(\frac{x-\mu}{\sigma}\right)^{2}},-\infty<x<\infty \text {. }
$$

The probability function of $T=e^{x}$ is given by

$$
f(t)=\frac{1}{\sigma t \sqrt{2 \pi}} e^{-\frac{1}{2}\left(\frac{t-\mu}{\sigma}\right)^{2}}, t>0 .
$$

Cumulative distribution function:

$$
F(t)=\Phi\left(\frac{\log t-\mu}{\sigma}\right)
$$

Survival function:

$$
S(t)=1-\Phi\left(\frac{\log t-\mu}{\sigma}\right) .
$$

\subsubsection{Applications of the lognormal distribution}

The following are some of the areas we could apply the lognormal distribution: helps to analyze time spent by active users on online activity; helps in the income analysis of a certain population; helps in decoding the stock market fluctuations; find the length of a social media comment made by a user; calculate the maximum value of annual rainfall; and estimate the size of a biological specimen.

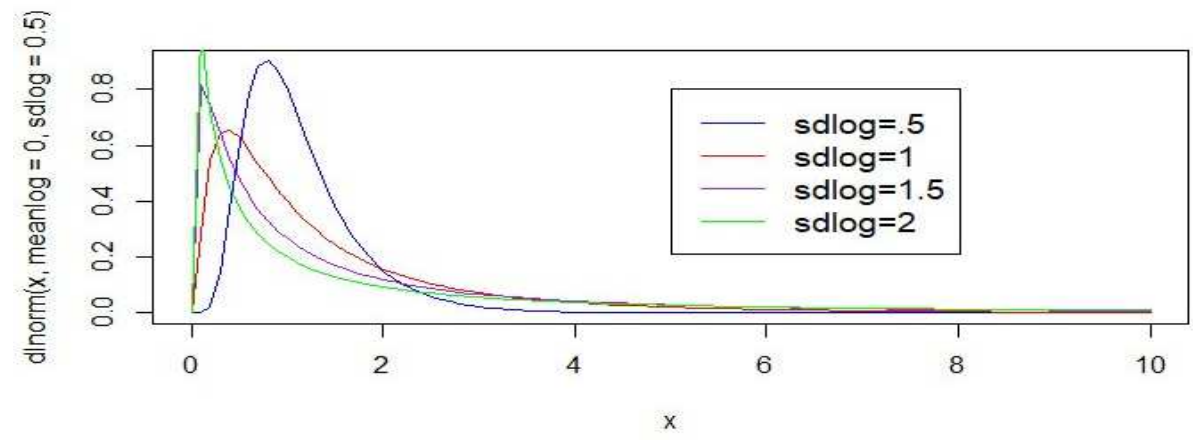

Figure 3: Plot of a probability density function for a log normal distribution with mean $=0$ and standard deviation $=1$ (on a log scale) where the $\mathrm{x}$-axis of the plot ranges from 0 to 10 . 


\subsection{The reliability function}

The most frequently used function in life data analysis and reliability engineering is the reliability function. This function gives the probability of an item operating for a certain amount of time without failure. As such, the reliability function is a function of time, in that every reliability value has an associated time value. Reliability engineering uses statistics to plan maintenance, determine the life-cycle cost, forecast failures, and determine warranty periods for products. The reliability function is commonly denoted as $R(t)$ and is complement to the cumulative distribution function $R(t)=1-F(t)$. The usage of the terminologies survival function and reliability function are interchangeable [11]. Explanations and notations under the exponential, Weibull and the lognormal distributions follow: The reliability function represents the reliability for a mission of time $t$, starting at age 0 ; the conditional reliability function represents the reliability for a mission of time $t$ duration having already successfully accumulated $T$ hours of operation up to the start of the new mission. The reliability life function represents the estimated time at which time the reliability value will be equal to a specified goal.

\subsubsection{For the exponential distribution}

Reliability function: $R(t)=e^{-\lambda t}$.

Exponential conditional reliability function: $R(t)=\frac{R(T+t)}{R(T)}=e^{-\lambda t}$.

Exponential reliable life for desired reliability goal $\left(t_{i}\right): R\left(t_{l}\right)=e^{-\lambda\left(t_{i}-\gamma\right)} \cdot \gamma$ is the location parameter.

\subsubsection{For the Weibull distribution}

Reliability function: $R(t)=e^{-\left(\frac{t-\lambda}{\alpha}\right)^{\beta}}$.

Weibull conditional reliability function: $R(t)=\frac{R(T+t)}{R(T)}=\frac{e^{-\left(\frac{T+t-\lambda}{\alpha}\right)^{\beta}}}{e^{-\left(\frac{t-\lambda}{\alpha}\right)^{\beta}}}$.

Weibull reliable life for desired reliability goal: $T_{l}=\lambda+\alpha\{-\ln (R)\}^{\frac{1}{\beta}}$.

\subsubsection{For the lognormal distribution}

Reliability function: $R(t)=\int_{t}^{\infty} f(x) d x$ or $\int_{\lim t}^{\infty} \frac{1}{\sigma^{t} \sqrt{2 \pi}} e^{-\frac{1}{2}\left(\frac{x-\mu}{\sigma^{t}}\right)^{2}} d x$. 


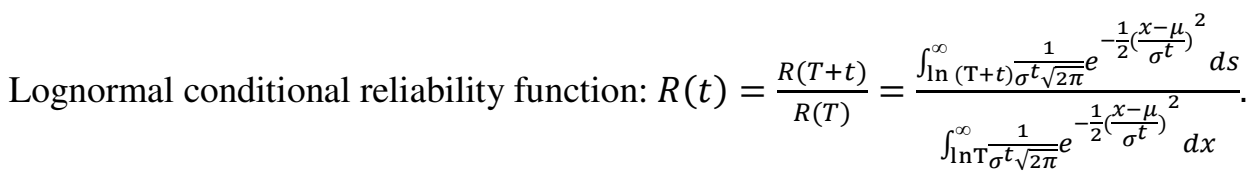

Lognormal reliable life for desired reliability goal: $R(t)=\int_{\operatorname{lnt}}^{\infty} \frac{1}{\sigma^{t} \sqrt{2 \pi}} e^{-\frac{1}{2}\left(\frac{x-\mu}{\sigma^{t}}\right)^{2}} d x$.

\section{Trends in the Application of the Frequentist and Bayesian Inferences in Failure Time Distributions}

Reference [16] conducted a study in which they used type II censored data, the study was left open at the beginning and allowed to run until a pre-specified fraction $\frac{r}{n}$ of the $n$ items had "failed". They denoted the random samples with the ordered values $T_{1}, T_{2}, \ldots, T_{n}$. The study was terminated after the $r^{t h}$ failure time had occurred, $r<n$. The amount of censored data was $n-r$. They defined the scheme of type II censored data as $y_{1}, y_{2}, \ldots, y_{r}$ with $y \leq z$ and $r<n$, again $z_{r+1}, z_{r+2} \ldots z_{n}$

$$
\sum_{i=1}^{n} x_{i}=\sum_{j=1}^{r} y_{j}+\sum_{k=r+1}^{n} z_{k}
$$

Where,

$x_{i}$ : complete data; $y_{i}:$ uncensored data; $z_{k}:$ censored data; $n:$ amount of complete data; $r$ : amount of uncensored data; $n-r:$ amount of censored data.

The one parameter shape parameter $(\beta)$ Weibull distribution for theprobability density function (PDF), cumulative distribution function (CDF), and survival functions were given respectively as Equations (28), (29) and (30):

$$
\begin{aligned}
& f(x)=\beta(x)^{\beta-1} \exp (-x)^{\beta}, x>0, \beta>0 \\
& F(x)=1-\exp (-x)^{\beta}, x>0, \beta>0 \\
& S(x)=\exp (-x)^{\beta}, x>0, \beta>0
\end{aligned}
$$

[17-19] used the maximum likelihood approach in their analysis of failure time data connected to Equations (28), (29) and (30), while [20] used the Bayesian method. According to [21] the Weibull distribution, which is used for modeling survival data, is embedded in a larger family obtained by introducing an additional shape parameter. This generalized family does not only contain distributions with unimodal and bathtub hazard shapes, but also allows for a broader class of monotone hazard rates. Furthermore, the distributions in this family were analytically tractable and computationally manageable. 
[22] encourages practitioners to use the Weibull distribution by furnishing historical background and parallel development of the essential formulas (cumulative distribution functions, probability density functions, hazard rates, and parameter estimates) for each of five formulations. [23] supported the fact that the exponential and Weibull distributions were the most frequently used parametric models in survival analysis, with the importance of the exponential distribution stemming from the lack of memory property and constant hazard rate function. It also emerged that the Weibull distribution contained the exponential distribution and constituted a more general model for the survival analysis since it does not assume a constant hazard rate. They combined the Poisson distribution to the exponential and Weibull distributions to develop an extended version of these distributions. The new distribution could be seen as the distribution of first order statistic of random sample which could be used as an approximation to the lifetimes of patients who were subject to the same disease.

Reference [24] indicated that one of the ways we could analyze survival time function was to estimate the value of the distribution parameters. He was silent on the method to adopt. They asserted that parameter estimation could be approached in two ways: The classical (frequentist) and Bayesian approaches. They averred that in the classical approach, the procedure was carried out by looking at the performance of all possible random samples at a particular point in time, that means, sample information obtained previously in the past were ignored. The Bayesian approach was different, it viewed parameters as variables that described initial knowledge about parameters before observations were made and represented in a distribution, called prior distribution. In the Bayesian approach the information from the prior data and information of the parameters were combined. By merging the two information, they obtained a posterior data that could be used to get the value of the estimated parameters. The posterior distribution expressed a person's degree of confidence about a parameter after the sample had been observed. [25] proposed Bayesian survival estimator for Weibull distribution with censored data. [26] also proposed Bayesian approach with Jeffrey prior in estimating of Weibull parameter using censored data. [27] used Bayesian Weibull survival model in the prediction of patients' survival with lymphoma disease based on the gene expression. [26] in their studies of the robustness of the various probability distributions concluded that, the Weibull distribution stood out tall. They also noted that there had been a contention between the frequentist and the Bayesian approaches. In their quest to settle on one of the approaches, they explored and compared the performance of the Maximum likelihood and Bayesian methods using Jeffrey prior and the extension of Jeffrey prior 
information for estimating the survival function of Weibull distributions with right censored data. Their conclusion was that the estimate of the survival function of maximum likelihood was the best compared with the others especially when the value of extension of Jeffrey prior was 0.4 .

Reference [28] underscored the fact that the Bayes posterior was a probability distribution function for which reason we could use credible interval (CI) quantiles instead of confidence intervals. He noted that there was a more intuitive interpretation of credible intervals than confidence intervals and that in either case, the differences between frequentist and Bayesian methods became negligible as the sample size increased. He however observed that when the data sets were small, the differences could be significant, with the Bayesian interval estimates becoming narrower than the frequentist methods. [29] has observed that contrary to the frequentist approach to statistical inference, the Bayesian approach allows the researcher to determine the probability of the null hypothesis, or a range of values within which the true value laid with a predetermined level of probability. [30] asserted that the frequentist framework, despite its popularity, had several serious deficiencies. They showed how the Bayesian framework was both flexible and principled, and concluded that the field of psychology could gain a lot by moving from the frequentist framework to the Bayesian framework for statistical inference. [30] highlighted on the reasons why the frequentist framework was a less-than-ideal method for statistical inference. Among other limitations, they unraveled that the most fundamental limitation of standard frequentist inference was that, it did not condition on the observed data. The resulting paradoxes did sparkle a philosophical debate that statistical practitioners had conveniently ignored. What they could not easily ignore was the practical limitations of frequentist inference, such as its restriction to nested model comparisons. They further corroborated with other schools of thought which highlighted the theoretical and practical advantages of a Bayesian analysis. They noted that from a theoretical perspective, Bayesian inference was principled and prescriptive, when contrasted with the frequentist inference. They crowned the discussion with a note that the Bayesian inference was becoming more and more attractive, because of recent advances in computational methodology like the Markov chain Monte Carlo. After a considerable study of the Bayesian and frequentist approach to analyzing failure time data. In [31], the authors concluded that the Bayesian analysis provided a clear and intuitive method to address reliability failure time analysis, especially when frequentist methods fell short. 


\subsection{Discriminating between the Weibull and the lognormal distribution}

In reliability analysis, both the Weibull and the lognormal distributions could be analyzed using the observed data logarithms. While the Weibull data logarithm is skewed, the lognormal data logarithm is symmetrical. [32] used the following methods to discriminate between the distributions: the coefficients of variation $(\mathrm{CV})$; the standard deviation of the data logarithms; the percentile position of the mean of the data logarithm; and the cumulated logarithm dispersion before and after the mean. The efficiency of the method was duly tested. A stress-strength data set was used for the analysis. The first data set corresponded to the stress load in a machine that used a plunger to press a shaft into a bushing. The second data set corresponded to the strength of the plunger when it was subjected to compression loads. The following conclusions were drawn: The stress data followed a lognormal distribution, while the strength data followed a Weibull distribution, therefore for the stress-strength analysis the lognormal-Weibull combination was used. [33] embarked on a research journey to discriminate between the Weibull and the log-normal distribution. Based on historical antecedent, they settled on the use of ratio of the maximum likelihood (RML). In their concluding remarks they averred that though each of the distributions has great applications, none of them produced a good fit. [34] examined the use of Kullback-Leibler Divergence (KLD) in discriminating between the Weibull and Log-Normal distribution. Attention towards the discrimination between the two distributions was done due to increasing applications of lifetime models. Two reasons why the researcher shifted from the use of the ratio of maximized likelihood (RML) test statistic were as follows: KLD incorporates information contained in both models; all parameters play important roles in the test statistic. Two cases were subjected to hypothesis testing: Case one (1); $H_{0}$ : $\log$ normal vs $H_{1}=$ Weibull. The decision criteriumwas to reject $H_{0}$, if KLD was greater than a chosen critical value at a level of significance $\alpha=20 \%$. In the final analysis, the authors could not reject the lognormal in favour of the Weibull distribution model. Case two (2); $H_{0}$ : Weibull vs $H_{1}=$ $\log$ normal. The decision criterium was to reject $H_{0}$, if KLD was greater than a chosen critical value at a level of significance $\alpha=20 \%$. In the final analysis, the authors could not reject the Weibull in favour of the lognormal distribution model.

Reference [35] undertook a study where the ratio of maximized likelihood (RML) and Minimized Kullback-Leibler Divergence (RMKLD) methods were discussed for discrimination between log-normal and Weibull distributions. The progressive Type II right censored sample was considered. The probability of correct selections was 
simulated and compared to investigate the performance of the procedures for different censoring schemes and parameter settings. Discrimination procedures were performed to decide whether the data came from a Weibull or a Lognormal distribution. The test criteria for the rejection/selection of the models were as follows: Select Lognormal if $R M L>0$. Select the Weibull if $R M K L D>0$. Using the $\mathrm{R}$ code with non-linear minimization command, ML estimates of lognormal $\{\operatorname{scale}(\mu)$, shape $(\sigma)$ parameters were obtained as follows: $\mu=4.3079, \sigma=0.5886$, the ML estimates for the Weibull distribution $\quad\{\operatorname{scale}(\alpha)$, shape $(\beta)\} \quad$ parameters were $\alpha=2.211, \beta=95.3497$. Test statistics revealed that the $R M L=0.345>0$. Since the RML was greater than zero, the lognormal distribution was selected. Again, since the $R M K L D=0.6028>0$, the Weibull distribution was selected.

\section{Discussions}

References [21,22] encouraged researchers to use the Weibull distribution by preparing historical background and development of the essential formulas. [23] supported the fact that the exponential and Weibull distributions were the most frequently used parametric models in survival analysis, with the importance of the exponential distribution stemming from the lack of memory property and constant hazard rate function. It also emerged that the Weibull distribution contained the exponential distribution and constituted a more general model for the survival analysis since it does not assume a constant hazard rate. [24] showed that one of the ways we could analyze survival time function was to estimate the value of the distribution parameters, which could be achieved in two ways: The classical (frequentist) and Bayesian approaches. They averred that in the classical approach, the procedure was carried out by looking at the performance of all possible random samples at a particular point in time, that means, the sample information obtained previously in the past were ignored. The Bayesian approach was different, it viewed parameters as variables that described initial knowledge about parameters before observations were made and represented in a distribution, called prior distribution. In the Bayesian approach the information from the prior data and from the parameters were combined, by merging the two information, a posterior distribution was obtained (The posterior distribution expresses a person's degree of confidence about a parameter after the sample has been observed) which is used to get the value of the estimated parameters. [25] proposed Bayesian survival estimator for Weibull distribution with censored data. [26] also proposed Bayesian approach with Jeffrey prior in estimating of Weibull parameter using censored data. [24,27] used Bayesian Weibull survival model 
in the prediction of patients' survival with lymphoma disease based on the gene expression.

Table 1: Concise description of the log-normal and Weibull probability distribution functions.

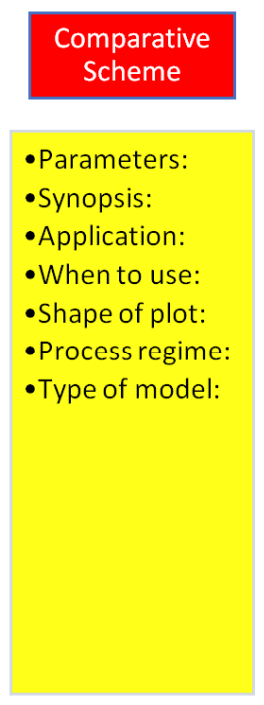

Log normal Distribution

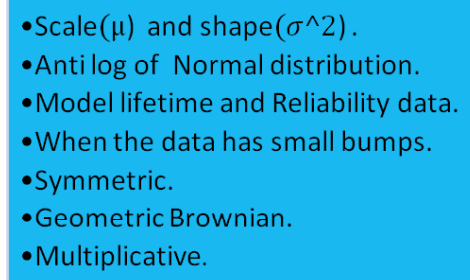

Weibull Distribution

- location $(\lambda)$, shape $(\beta)$ and scale $(\alpha)$

- Extention of the exponential distribution.

- Model lifetime and Poisson processes.

-When exponential distribution does to fit.

- Skewed.

-Non-homgenous Poisson.

-Additive.

Reference [26] in their studies of the robustness of the various probability distributions concluded that, the Weibull distribution stood out tall. [28] noted that the differences between the frequentist and Bayesian methods become negligible as the sample size increases. He observed that when the data sets were small, the differences could be significant, with the Bayesian interval estimates becoming narrower than the frequentist methods. [29] observed that contrary to the frequentist approach to statistical inference, the Bayesian approach allows the researcher to determine the probability of the null hypothesis, or a range of values within which the true value lies with a predetermined level of probability. [30] asserted that the frequentist framework, despite its popularity, had several serious deficiencies. They showed how the Bayesian framework was both flexible and principled, and concluded that the field of psychology could gain a lot by moving away from the use of the frequentist framework to the Bayesian framework for statistical inference. [31] after a considerable study of the Bayesian and frequentist approach to analyzing failure time data, concluded that the Bayesian analysis provided a clear and intuitive method to addressing reliability failure time analysis, especially when frequentist methods fell short. The discriminating study 
between the Weibull and lognormal distribution carried out by References [32-35], revealed that each of the distributions had a peculiar application. In the final analysis, the authors could not reject the Weibull in favour of the lognormal distribution model, neither could they reject the lognormal distribution in favour of the Weibull. For the stress-strength analysis the lognormal-Weibull combination was used.

Figure 1 shows the plot of an exponential distribution, it could be seen from the plot that the higher the rate, the gentler the slope of the curve and vice versa. In Figure 2, which showcased the plot of the Weibull distribution. The plot revealed that the bigger the shape parameter, the more crowning the curve was and vice versa. It was again observed that, when the shape parameter $=1$ and scale parameter $=1$, the curve becomes an exponential distribution (compare Figure 1 and Figure 2). Moreover, when the shape parameter $=2$, the Weibull distribution assumes another name called the Rayleigh distribution which turns out to be a theoretical probability model. This model is widely used in communications theory to model multiple paths of dense scattered signals reaching a receiver. In the physical sciences, Rayleigh distribution is used to model wind speed, wave heights and sound or light radiation. In engineering, it is used to measure the lifetime of an object, where the lifetime depends on the object's age. Two cases were observed in the plot of the Weibull distribution: Case one, the shape parameter was varied with the scale parameter kept constant; case two, scale parameter was varied with the shape parameter kept constant. It could be inferred from these two cases that the scale parameter does not affect the peak of the curve, but rather the spread of the curve. For shape parameter $(\beta<1)$, the reliability decreases sharply very early in the respective product's life, and then slowly thereafter. For shape $(\beta)$ parameters greater than 1 , the initial drop in reliability was small, and then the reliability drops relatively sharply at some point later in time. The point where all curves intersect is called the characteristic life: regardless of the shape parameter, 63.2 percent of the population will have failed at or before the point of intersection, that is $\{R(t)=1-0.632=0.368\}$. This point in time is also equal to the respective scale parameter $\alpha$ of the two-parameter Weibull distribution. For $0<\beta \leq 1$, as $x \rightarrow 0, f(x) \rightarrow \infty$ and as $x \rightarrow 0, f(x) \rightarrow 0$. The function $f(x)$ decreases monotonically and is convex, the mode is non-existent. Figure 3 displays the plot of the probability density function for a log normal distribution with mean $=0$ and standard deviation $=1$ (on a log scale), the plot shows that the smaller the standard deviation of the log of the shape parameters, the near normal the distribution becomes and vice versa. 
Table 2 gives a vivid description and attributes of the lognormal and Weibull distribution functions.

The two distributions, Weibull and the lognormal distribution operate on different statistical processes and therefore cannot be used interchangeably, howbeit they can be used together. In reliability analysis, a wrong selection of the appropriate distribution will either underestimate or overestimate the reliability values. The adage which says that in the mouth of two or three witnesses there is safety can be conveniently applied here. From the researches done by [32-35], it can be concluded that both the Weibull and lognormal distributions could be applied in lifetime data analysis.

\section{Conclusion and Recommendation}

The literature is replete with information about the dominance of the frequentist inference in certain health fields including experimental psychology. Standing on the protocol of [36]. It is observed that with the frequentist approach, the parameter is unknown, but fixed quantity which rely only on the information coming from the sampling data for inference. In other words, the researcher does not take into consideration the prior belief on the parameter. In contrast, the Bayesian approach, considers the parameter as a random variable with a certain probability distribution, known in the literature as the prior distribution, the prior distribution represents the researchers belief before the data is observed. The prior distribution could be updated on the basis of the likelihood function through the Bayes' theorem into a posterior distribution which summarizes the information in both the prior distribution and in the data.

Cruising through the few literature on the topic under discussion, it is noted that out of the three failure time distributions, that is, Exponential, Weibull and Log-normal distributions, the Weibull distribution and the lognormal distributions have specific statistical application and therefore care must be taking in selecting the appropriate distribution, moreover, with respect to the frequentist approach and the Bayesian approach to conducting statistical inferences, it came out strongly that it was high time psychologist who have adopted the use of the frequentist framework moved away from it and started using the Bayesian approach. It is therefore concluded and recommended that the Bayesian lognormal-Weibull distribution be used since it is more robust than other distribution. 


\section{References}

[1] S. Selvin, Survival Analysis for Epidemiology and Medical Research: A Practical Guide, Cambridge University Press, New York, 2008.

[2] S. F. Morris, Confidence Limits - Exponential Distribution, Reliability Analytics Corporation, 2017

[3] J. Kensler, and L. Cortes, Interpreting confidence intervals, Scientific Test and Analysis Techniques Center of Excellence (STAT COE), 24 December 2014.

[4] D. R. Cox, Some problems connected with statistical inference, The Annals of Mathematical Statistics 29 (1958), 357-372. https://doi.org/10.1214/aoms/1177706618

[5] R. T. Cox, Probability, frequency and reasonable expectation, The American Journal of Physics 14 (1946), 1-13. https://doi.org/10.1119/1.1990764

[6] A. P. Dawid, Statistical theory: The prequential approach, Journal of the Royal Statistical Society A 147 (1984), 278-292. https://doi.org/10.2307/2981683

[7] B. De Finetti, Theory of Probability, Vols. 1 and 2, Wiley, New York, 1974. https://doi.org/10.1007/978-94-010-2288-0_1

[8] S. Dennis and M. S. Humphreys, A context noise model of episodic word recognition, Psychological Review 108 (2001), 452-477.

https://doi.org/10.1037/0033-295X.108.2.452

[9] J. O. Berger, Could Fisher, Jeffreys and Neyman have agreed on testing?, Statistical Science 18 (2003), 1-32. https://doi.org/10.1214/ss/1056397485

[10] R. Christensen, Testing Fisher, Neyman, Pearson, and Bayes, The American Statistician 59 (2005), 121-126. https://doi.org/10.1198/000313005X20871

[11] E. T. Lee, Statistical Methods for Survival Data, Belmont, CA: Wadsworth, Inc., 1980.

[12] J. D. Kalbfleisch and R. L. Prentice, The Statistical Analysis of Failure Time data, New York: John Wiley and Sons, 1980.

[13] J. F. Lawless, Statistical Models and Methods for Lifetime Data, New York: John Wiley and Sons, 1982.

[14] T. Vaughan, In search of the memoryless property, Conference: Proceedings of the 2008 Winter Simulation Conference, Global Gateway to Discovery, WSC 2008, InterContinental Hotel, Miami, Florida, USA, December 7-10, 2008. https://doi.org/10.1109/WSC.2008.4736369

[15] H.-P. Blossfeld and G. Rohwer, Techniques of Event History Modeling, Mahwah, NJ: Lawrence Erlbaum Associates, 1995, pp. 667-694. 
[16] A. Kurniawan, N. Avicena and E. Ana, Estimation of the shape parameter of Weibull distribution based on type ii censored data using EM algorithm, AIP Conference Proceedings 2264 (2020), 030011. https://doi.org/10.1063/5.0025681

[17] H. Fei, F. Kong and Y. Tang, Estimation for two-parameter Weibull distribution and extreme-value distribution under multiply type-II censoring, Journal Communication in Statistics-Theory and Methods 24 (1995), 2087-2104.

https://doi.org/10.1080/03610929508831604

[18] F. G. Akgul and B. Senoglu, Estimation of stress-strength reliability for Weibull distribution based on type-II right censored ranked set sampling data, Pakistan Journal of Statistics and Operation Research 14 (2018), 781-806.

https://doi.org/10.18187/pjsor.v14i4.2239

[19] V. Sujatha, R. Ravanan and M. Ramakrishnan, Point and interval estimates of Weibull distribution to progressively type ii censored data by order statistics approach, International Journal of Recent Technology and Engineering 6 (2010), 370-374.

[20] H. Panahi and S. Asadi, Estimation of the Weibull distribution based on type-II censored samples, Journal Applied Mathematical Statistics 5 (2011), 2549-2558.

[21] G. S. Mudholkar, D. K. Srivastava and G. D. Kollia, A generalization of the Weibull distribution with application to the analysis of survival data, Journal of the American Statistical Association 91 (1996), 1575-1583.

https://doi.org/10.1080/01621459.1996.10476725

[22] A. J. Hallinan, Jr., A review of the Weibull distribution, Journal of Quality Technology 25 (1993), 85-93. https://doi.org/10.1080/00224065.1993.11979431

[23] S. Çelebioğlu, On an extension of the exponential and Weibull distributions, SDU Journal of Science (E-Journal) 5 (2010), 137-146.

[24] S. A. Thamrin, M. N. Zoraida, A. K. Jaya and Ansariadi, Performance of Bayesian using conjugate prior estimator for Weibull right censored survival data, Asian Journal of Scientific Research 11 (2018), 376-382. https://doi.org/10.3923/ajsr.2018.376.382

[25] A. O. M. Ahmed, N. A. Ibrahim, J. Arasan and M. B. Adam, Extension of Jeffreys's prior estimate for Weibull censored data using Lindley's approximation, Aust. J. Basic Applied Sci. 5 (2011), 884-889.

[26] A. O. M. Ahmed and N. A. Ibrahim, Bayesian survival estimator for Weibull distribution with censored data, J. Applied Sci. 11 (2011), 393-396.

https://doi.org/10.3923/jas.2011.393.396

[27] S. A. Thamrin, J. M. McGree and K. L. Mengersen, Bayesian Weibull survival model for 
gene expression data, in: Case Studies in Bayesian Statistical Modelling and Analysis, W.A. Shewhart, S.S. Wilks, C.L. Alston, K.L. Mengersen and A.N. Pettitt, eds., Wiley, New York, 2013, pp. 171-185. https://doi.org/10.1002/9781118394472.ch10

[28] M. Hamada, A. Wilson, C. S. Reese and H. Martz, Bayesian Reliability, New York: Springer, 2008. https://doi.org/10.1007/978-0-387-77950-8

[29] L. D. Walker, Hypothesis Tests and Proofs, Encyclopedia of Social Measurement (2005), 265-270. https://doi.org/10.1016/B0-12-369398-5/00019-0

[30] E. J. Wagenmakers, M. Lee, T. Lodewyckx and G. J. Iverson, Bayesian versus frequentist inference, in: Bayesian Evaluation of Informative Hypotheses, Springer, New York, 2008. https://doi.org/10.1007/978-0-387-09612-4_9

[31] M. Harman, Practical Bayesian Analysis of Failure Time Data, STAT COE-Report-102017.

https://www.afit.edu/stat/statcoe_files/Practical_Bayesian_Analysis_for_Failure_Time_D ata_Best_Practice.pdf

[32] J. F. Ortiz-Yañez and M. R. Piña-Monarrez, Discrimination between the lognormal and Weibull distributions by using multiple linear regression, DYNA 85 (2018), 9-18. https://doi.org/10.15446/dyna.v85n205.66658

[33] D. Kundu and A. Manglick, Discriminating between the Weibull and log-normal distributions, Naval Research Logistics 51 (2004), 893-905. https://doi.org/10.1002/nav.20029

[34] A. A. Bromideh, Discriminating between Weibull and log-normal distributions based on Kullback-Leibler divergence, Istanbul University Econometrics and Statistics e-Journal 16 (2012), 44-54.

[35] Coşkun Kuş, A. Pekgör and I. Kinaci, Discriminating between the lognormal and Weibull distributions under progressive censoring, Cumhuriyet Science Journal 40 (2019), 493504. https://doi.org/10.17776/csj.470148

[36] F. Bartolucci and L. Scrucca, Point estimation methods with applications to item response theory models, International Encyclopedia of Education (Third Edition) (2010), 366-373. https://doi.org/10.1016/B978-0-08-044894-7.01376-2

\footnotetext{
This is an open access article distributed under the terms of the Creative Commons Attribution License (http://creativecommons.org/licenses/by/4.0/), which permits unrestricted, use, distribution and reproduction in any medium, or format for any purpose, even commercially provided the work is properly cited.
} 